Revue des patrimoines

13 | 2010

Art rupestre : la 3D un outil de médiation du réel invisible?

\title{
Restauration virtuelle de l'art pariétal paléolithique : le cas de la grotte de Marsoulas
}

Carole Fritz, Gilles Tosello, Marc Azéma, Olivier Moreau, Guy Perazio et José Péral

\section{(2) OpenEdition}

Journals

Édition électronique

URL : http://journals.openedition.org/insitu/6774

DOI : $10.4000 /$ insitu. 6774

ISSN : 1630-7305

Éditeur

Ministère de la culture

Référence électronique

Carole Fritz, Gilles Tosello, Marc Azéma, Olivier Moreau, Guy Perazio et José Péral, « Restauration virtuelle de l'art pariétal paléolithique : le cas de la grotte de Marsoulas », In Situ [En ligne], 13 | 2010, mis en ligne le 16 avril 2012, consulté le 19 avril 2019. URL : http://journals.openedition.org/ insitu/6774 ; DOI : 10.4000/insitu.6774

Ce document a été généré automatiquement le 19 avril 2019.

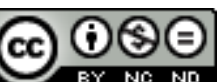

In Situ Revues des patrimoines est mis à disposition selon les termes de la licence Creative Commons Attribution - Pas d'Utilisation Commerciale - Pas de Modification 4.0 International. 


\title{
Restauration virtuelle de l'art pariétal paléolithique : le cas de la grotte de Marsoulas
}

\author{
Carole Fritz, Gilles Tosello, Marc Azéma, Olivier Moreau, Guy Perazio et \\ José Péral
}

\section{Introduction}

Depuis quelques années, les techniques de scanner 3D sont appliquées dans des grottes et abris préhistoriques ornés ${ }^{1}$ avec un succès croissant car elles offrent une vision en volume de l'art pariétal, attrayante et accessible à tous. Jusqu'à présent, les technologies 3D ont été plutôt utilisées pour la phase finale de l'étude, synthétisant l'ensemble des données et proposant une vision d'ensemble du site. Au-delà, elles ouvrent de vastes champs d'expérimentation et autorisent la reconstitution de monuments disparus ou parvenus jusqu'à notre époque à l'état de vestiges. Appliquée à l'art paléolithique, l'imagerie 3D peut même aider à restaurer les parois des cavernes atteintes par l'usure du temps et le vandalisme des hommes. Datée de 15000 ans, la grotte pyrénéenne de Marsoulas (HauteGaronne) convenait parfaitement à ce type d'expérimentation plutôt ambitieuse. À notre connaissance, une telle «restauration virtuelle» d'une galerie ornée au Paléolithique n'avait jamais été tentée; cependant, cette première n'a pu être accomplie qu'en combinant la technologie 3D aux résultats de l'étude scientifique du site et du relevé des parois ornées.

\section{Une grotte au passé riche et mouvementé}


Figure 1

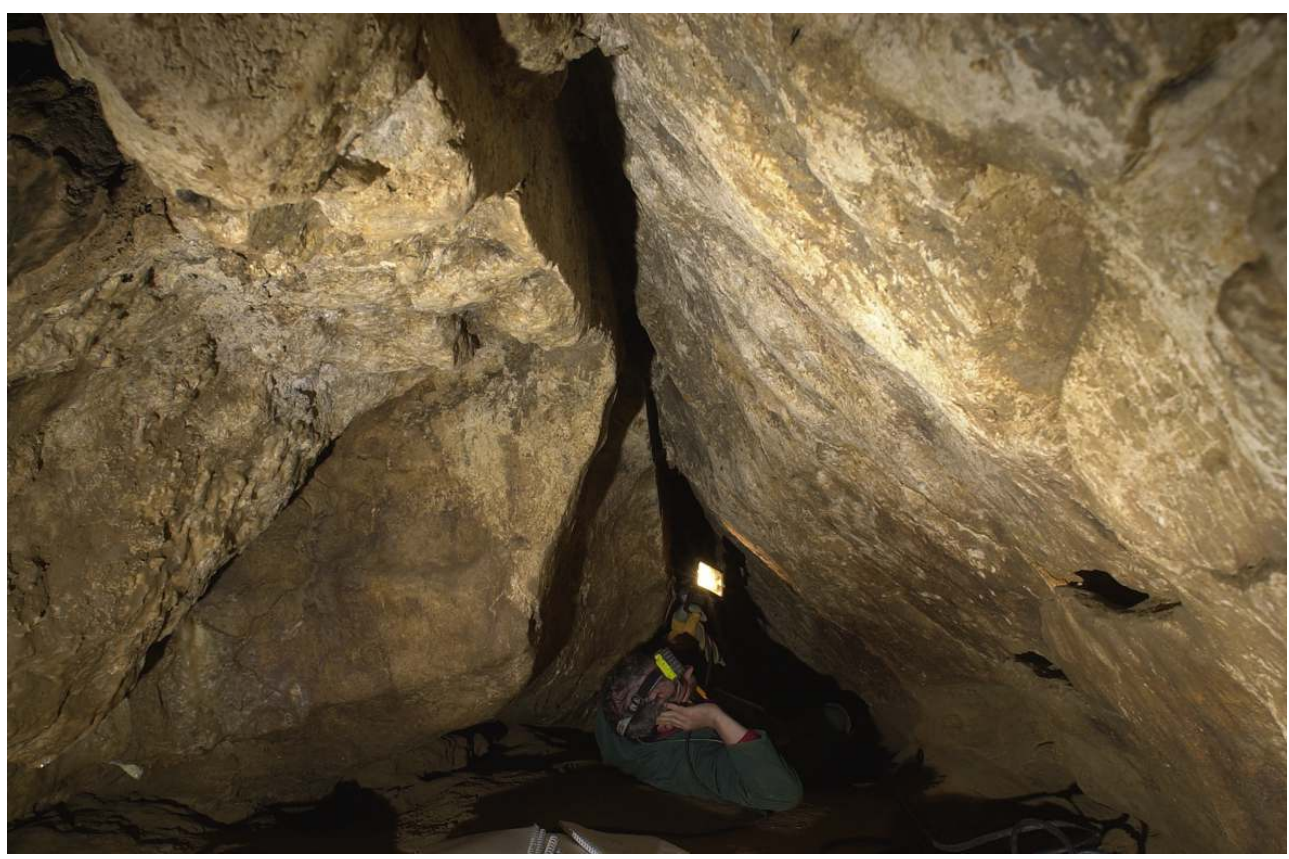

Grotte de Marsoulas, vue de la galerie à $41 \mathrm{~m}$ de l'entrée

Phot. Carole Fritz. (c) Ministère de la Culture et de la Communication, Carole Fritz

2 D'orientation nord-ouest/sud-est, la grotte s'ouvre sur le flanc sud d'un massif calcaire entaillé par un vallon au fond duquel serpente le petit ruisseau du Laouin. Comparée à ses prestigieuses voisines telles Labastide ou Niaux, Marsoulas est une modeste galerie rectiligne, étroite et longue d'une cinquantaine de mètres à peine. À 29 mètres de la porte, un resserrement brutal des parois et un abaissement de la voûte obligent le visiteur à s'accroupir puis à ramper (fig. $\mathbf{n}^{\circ} \mathbf{1}$ ). À 40 mètres, le sol s'incline suivant une pente abrupte tandis que la voûte conserve sa hauteur pendant une dizaine de mètres ; à partir de 44 mètres, on peut de nouveau se tenir debout jusqu'au lit actuel du ruisseau (50 mètres). Au-delà, la galerie se réduit et devient progressivement impénétrable.

3 En coupe, la grotte possède un profil triangulaire dissymétrique ( $3 \mathrm{~m}$ de large $\mathrm{x} 4 \mathrm{~m}$ de haut environ dans les parties les plus spacieuses) (fig. $\mathbf{n}^{\circ} \mathbf{2}$ ). La paroi droite nettement oblique s'incline jusqu'à former la voûte et vient s'appuyer sur la gauche, parfaitement verticale. L'art pariétal est présent sur toute la longueur du parcours, dès l'entrée et jusqu'au fond. 
Figure 2

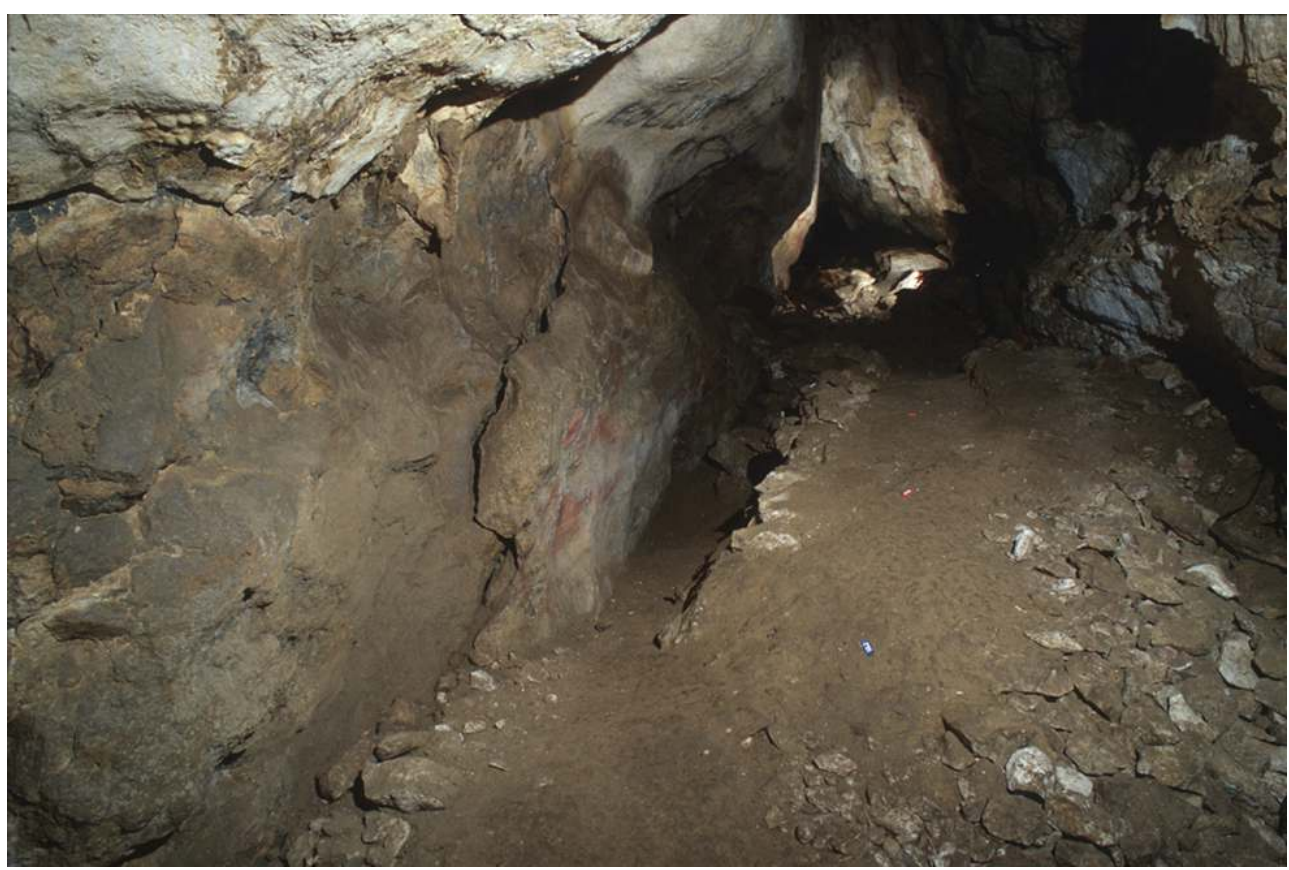

De l'entrée au fond, la galerie est rectiligne et sa section est triangulaire. À gauche, tranchée de fouilles anciennes

Phot. Carole Fritz. @ Ministère de la Culture et de la Communication, Carole Fritz

Première grotte à peintures préhistoriques signalée dans les Pyrénées, Marsoulas possède une longue histoire scientifique. La tranchée des fouilles ${ }^{2}$ de 1883-1884 est encore visible aujourd'hui, au pied de la paroi gauche entre 13 et $18 \mathrm{~m}$ de l'entrée. Outre un important matériel archéologique, le principal intérêt de cette excavation reste le dégagement de gravures et de peintures jusque-là recouvertes par le remplissage.

5 Marsoulas a joué un rôle important dans le débat animé qui, au tournant du siècle, opposait partisans et adversaires de l'existence d'un art «antédiluvien». En 1897, F. Regnault découvre les peintures du panneau principal et publie aussitôt une note accompagnée d'un croquis ${ }^{3}$. Cette notoriété a eu de lourdes conséquences pour la conservation du site, en y attirant des générations de visiteurs qui, par ignorance ou vandalisme, ont causé de graves dommages aux parois, partout où elles étaient à portée de main (fig. $\mathbf{n}^{\circ}$ 3). Aucune mesure de protection ne s'avérant entièrement efficace, la fermeture complète du site au public fut décidée en 1996. 
Figure 3

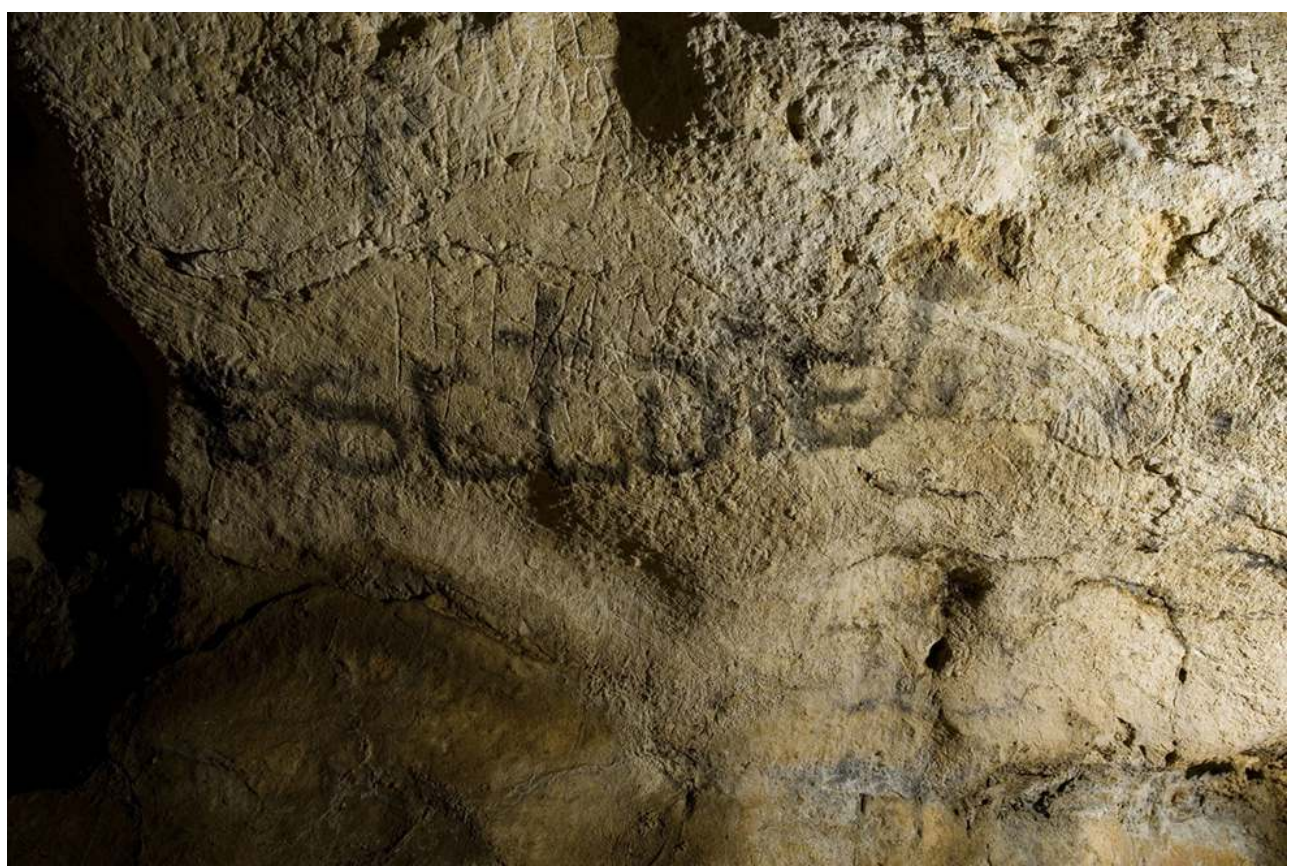

État actuel de la paroi gauche, entre 13 et 15 m de l'entrée. Les tracés préhistoriques sont recouverts par des graffitis

Phot. Carole Fritz. ( C Ministère de la Culture et de la Communication, Carole Fritz

6 Tout au long du $\mathrm{XX}^{\mathrm{e}}$ siècle, de nombreux chercheurs ont consacré des travaux à la cavité : É. Cartailhac, H. Breuil (qui réalisa des relevés mais n'en publia qu'une infime partie) ${ }^{45}, \mathrm{H}$. Bégouën, T. Russell et aussi L. Méroc, A. Leroi-Gourhan, A. Plénier (qui y consacra sa thèse ${ }^{6}$, D. Vialou, P. Foucher, S. Lacombe.... Pourtant, une bonne part de l'art pariétal restait inédite, constat qui nous conduisit à reprendre l'étude du site en $1998^{7}$. En s'appuyant sur les travaux de Breuil et Plénier, une révision complète des parois a été entreprise. Le contexte archéologique, important dans cette grotte qui fut aussi un habitat, est pris en compte. Par une collecte systématique des faits, on vise à replacer Marsoulas au sein des réseaux culturels magdaléniens en utilisant les analogies stylistiques et thématiques avec d'autres sites, proches ou lointains. Dans ce domaine, les résultats acquis sont déjà substantiels et relancent une problématique de définition des territoires, dans une perspective plus large de géographie humaine des groupes paléolithiques du Sud-Ouest européen ${ }^{8}$.

\section{Un art pariétal d'un style original}

7 À la fin de l'année 2006, l'inventaire des thèmes représentés décomptait plus de 220 animaux, humains et signes. Parmi eux, on relève de grands bisons ou chevaux peints en rouge et noir et soulignés en gravure ; l'un des bisons est couvert de points rouges, un autre de points noirs. Des signes sont assemblés sur le grand panneau peint de la paroi gauche : rectangle, nappe et lignes de points et de tirets, $\mathrm{T}$ inversés, grilles et surtout grands «barbelés » formés d'un axe et de traits obliques accolés... L'art de Marsoulas possède une profonde originalité stylistique, qui n'offre que peu d'équivalent dans les sites de la région pour la période considérée. 
Les parois possèdent des reliefs tourmentés, des creux, des fissures... que les artistes préhistoriques ont souvent cherché à intégrer à leurs œuvres pour mieux exprimer la troisième dimension. Sur certains panneaux, c'est même l'ensemble de la composition qui paraît régi par l'alternance de niches et de surfaces planes. Les dimensions des figures sont très variables; malgré l'exiguïté du lieu, on rencontre des dessins miniatures dissimulés dans les replis de la roche et de grands animaux (de 1,20 m à 2,20 m) peu lisibles car ils dépassent le champ visuel.

\section{Déchiffrer avant de « restaurer »...}

En raison de l'état de conservation médiocre, de la finesse et de la densité des gravures, des graffitis qui défigurent certains panneaux et compliquent la lecture, l'art pariétal de Marsoulas doit être déchiffré pour être vraiment perçu. Depuis 1998, l'essentiel du travail dans la grotte a donc été consacré à l'analyse et au relevé des parois.

Figure 4

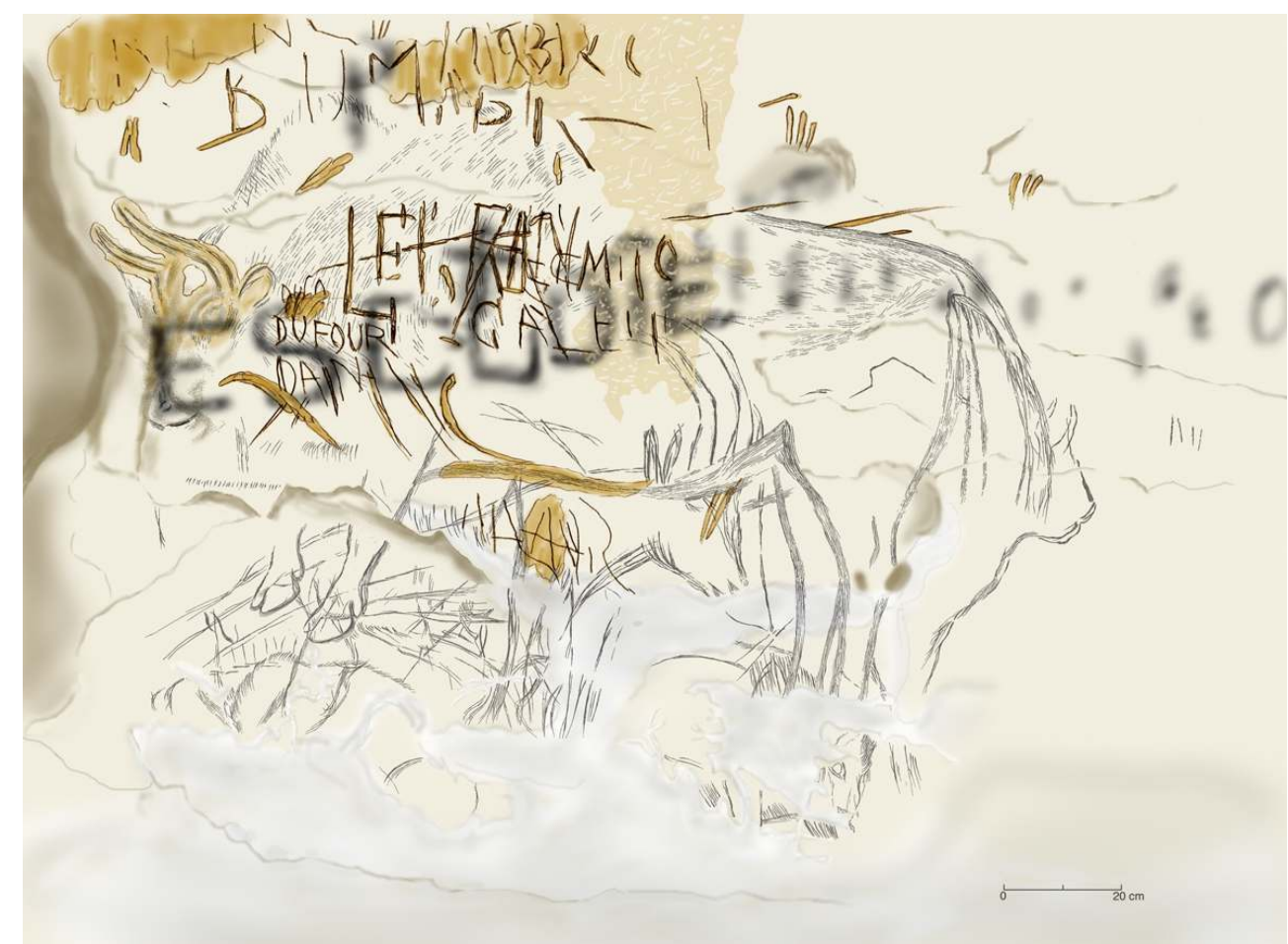

Relevé du panneau de la paroi gauche, entre 13 et $15 \mathrm{~m}$ de l'entrée (voir Figure $n^{\circ}$ 3). En gris, les gravures préhistoriques; en bistre, les destructions modernes

Doc. Carole Fritz, Gilles Tosello. (c) Ministère de la Culture et de la Communication, Carole Fritz, Gilles Tosello

Comme il est de règle aujourd'hui, ces opérations de recherche se déroulent en évitant tout contact avec les parois. L'étude de Marsoulas est donc menée sur la base d'une reproduction photographique des surfaces ornées qui sert de support aux observations et à l'enregistrement des données dans la grotte; ensuite, les relevés de terrain sont numérisés, replacés sur un fond qui imite la paroi rocheuse grâce à un traitement infographique. On parvient ainsi à une restitution synthétique des panneaux ${ }^{9}$. Les utilisations fréquentes de reliefs et l'insertion des compositions dans l'espace de la galerie 
nous ont incités depuis le début à consacrer beaucoup de temps et d'efforts à la restitution graphique des supports rocheux tout autant qu'à celle des figures.

Figure 5

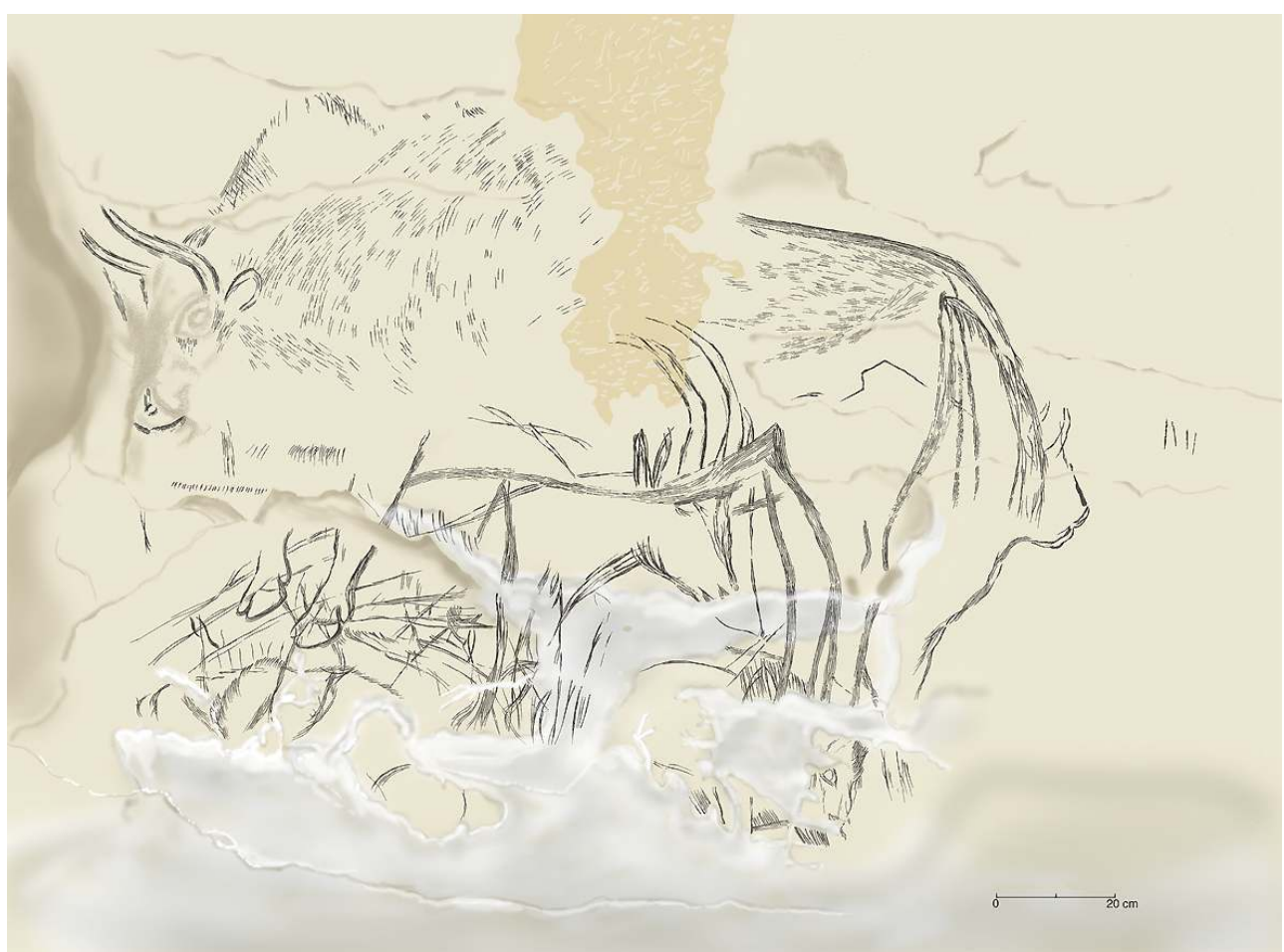

Relevé du panneau de la paroi gauche, entre 13 et $15 \mathrm{~m}$ de l'entrée (voir Figure $n^{\circ} 3$ et 4). L'élimination « virtuelle » des destructions modernes permet de proposer une restitution des gravures préhistoriques

Doc. Carole Fritz, Gilles Tosello. (c) Ministère de la Culture et de la Communication, Carole Fritz, Gilles Tosello

Les informations sont assemblées dans le logiciel par «calques" selon leur origine naturelle (concrétions, fissures, érosion...) ou anthropique. Dans ce dernier cas, on distingue ce qui est préhistorique (peinture rouge, noire, gravure fine ou profonde...) et ce qui ne l'est pas (graffitis, impacts divers, frottements...) (fig. $\mathbf{n}^{\circ}{ }^{4}$ ). En séparant les différents types d'informations, on peut facilement les sélectionner et par exemple, en visualisant les seuls tracés préhistoriques, suggérer l'aspect que présentait un panneau avant les altérations qui en perturbent la lecture aujourd'hui (fig. $\mathbf{n}^{\circ} \mathbf{5}$ ).

\section{Scanneur laser 3D et art pariétal}

Les performances des scanneurs laser 3D sont bien adaptées à l'étude de l'art préhistorique qui exige une résolution maximale en termes d'imagerie ${ }^{10}$. Dans les grottes ornées, la saisie se fait au moyen d'un scanneur laser de type Soisic ou Leica (la précision d'acquisition varie en fonction des modèles). Les points acquis par l'intermédiaire d'un laser balayant la paroi sont immédiatement visualisés sous la forme d'un nuage de points. Grâce à un logiciel, le nuage de points peut être ensuite triangulé (fig. $\left.\mathbf{n}^{\circ} \mathbf{6}\right)$ : les points sont reliés mathématiquement pour constituer un maillage de l'objet scanné, en l'occurrence ici la paroi. L'objet triangulé ou maillé doit être ensuite texturé. La 
corrélation («matching») des photos consiste à replacer chaque cliché (en haute résolution) selon l'axe focal de prise de vue afin de projeter l'image sur le maillage grâce à des points remarquables : c'est l'orthoprojection.

Figure 6

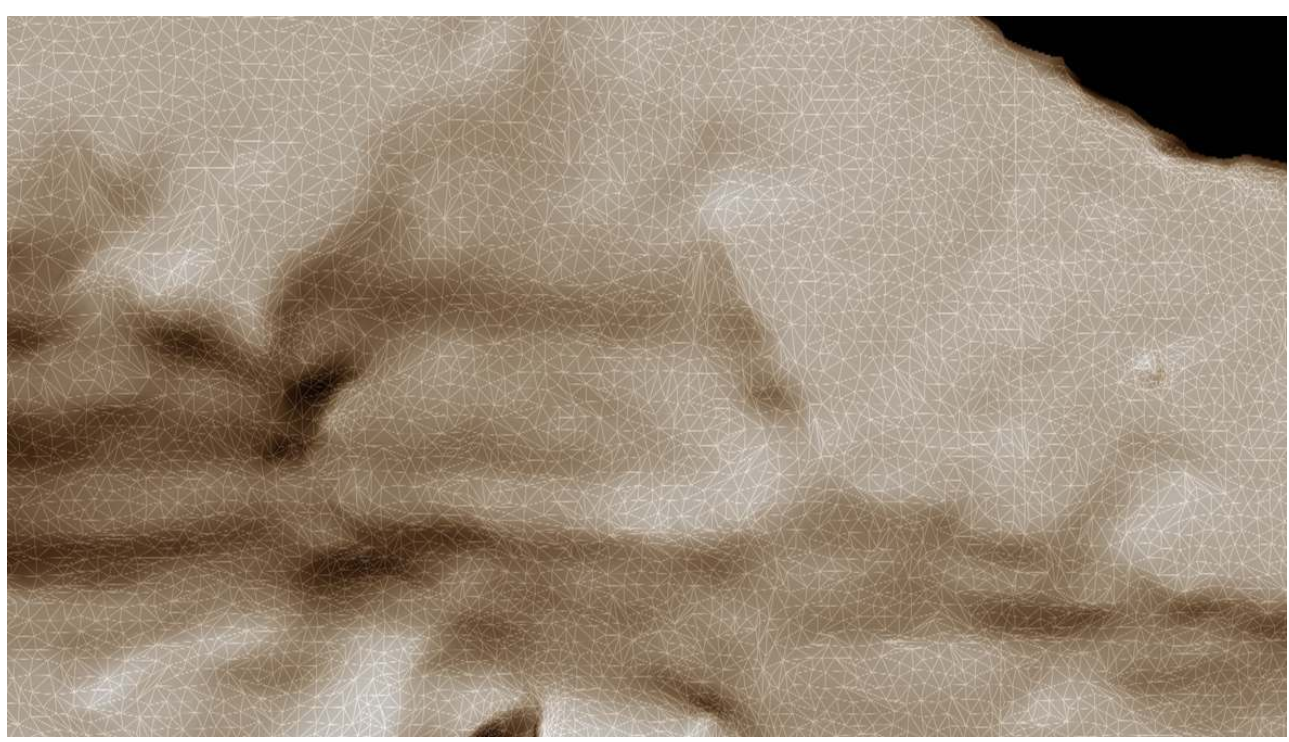

Modèle numérique triangulé et surfacé correspondant au centre du panneau principal (voir Figure $n$ $\circ$ 8)

Doc. Cabinet Perazio. ( ) Ministère de la Culture et de la Communication, Cabinet Perazio

L'application conjointe de la technologie 3D, de la photographie et du relevé sur calque apporte une évolution remarquable dans la traduction graphique des ensembles pariétaux mais aussi de leurs supports rocheux, par l'expression des volumes. Les ombres et lumières, les fissures qu'il fallait auparavant représenter par le dessin en « $2 \mathrm{D}$ » sont immédiatement lisibles grâce à la 3D; on peut aussi changer le point de vue, modifier la direction de l'éclairage pour améliorer la perception des œuvres dans leur contexte.

\section{La restauration virtuelle de la galerie ornée de Marsoulas}

À l'issue de trois missions de terrain ${ }^{11}$, la quasi-totalité de la grotte a été scannée en 3D avec une précision variable ( $1 \mathrm{~cm}$ pour les zones non ornées et $3 \mathrm{~mm}$ pour les autres) et dans un triple objectif :

- archiver des parois menacées par divers phénomènes naturels et dont la conservation à long terme est problématique. Un point de vue a pu être réalisé à l'extérieur, devant la porte afin de replacer la cavité dans la roche encaissante, ce qui permettra d'évaluer l'épaisseur de roche au-dessus de la grotte. À l'intérieur, l'accent a été mis sur le secteur du Grand Panneau peint, la principale concentration de peintures et gravures du site. Si cela est nécessaire, le nuage 3D permettra d'en faire un fac-similé de grande précision. Cependant, il reste des lacunes dans le nuage de points, dues aux difficultés d'installer certains types de lasers dans un espace contraignant et humide, problème résolu très récemment avec la dernière génération de machines; 
- tester l'apport de la 3D à la phase du relevé. Cette application est exposée dans un autre article de ce volume ${ }^{12}$;

- visualiser l'espace de la galerie et y replacer les panneaux gravés et peints au fur et à mesure de l'avancement de l'étude.

Une autre question nous préoccupait. La fermeture définitive du site aux visites touristiques (alors qu'il était accessible depuis la découverte) a créé une frustration auprès du public et nous cherchions depuis quelque temps à y remédier. Puisque les visiteurs ne pouvaient plus descendre dans la grotte, celle-ci pouvait-elle venir à eux ? L'idée d'une "visite virtuelle» fondée sur la 3D a donc fait son chemin mais elle a rapidement buté sur un constat décourageant: à quoi bon montrer les panneaux dans leur état de dégradation actuelle? N’allions-nous pas réduire la grotte à cette seule image négative ? C'est ainsi que le concept de « restauration virtuelle » s'est peu à peu imposé. Cette entreprise ambitieuse, qui n'avait jamais été tentée dans une grotte ornée, n'avait de sens que si elle s'appuyait sur une solide documentation scientifique, réunie grâce aux relevés et observations de terrain.

Figure 7

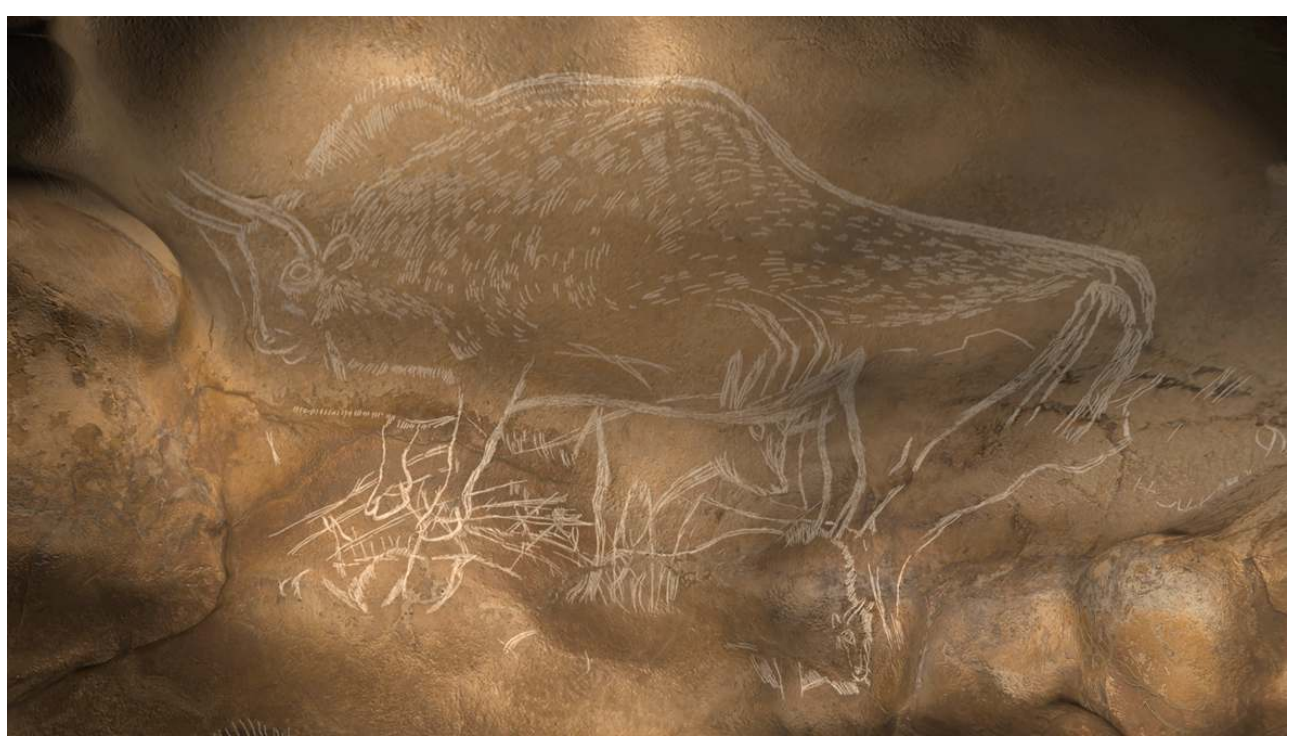

Relevé du panneau entre 13 et $15 \mathrm{~m}$ de l'entrée (voir Figure $n^{\circ} 3$ et 4) corrélé sur le modèle 3D texturé de la paroi, après élimination des destructions modernes

Doc. Carole Fritz, Gilles Tosello. (c) Ministère de la Culture et de la Communication, Carole Fritz, Gilles Tosello, Passé Simple, Cabinet Perazio

16 La réalisation d'un film documentaire sur Marsoulas a été le facteur déclenchant. En combinant les relevés graphiques en 2D, les techniques du scanneur laser et de l'infographie 3D, il a été possible de reconstituer une trentaine de mètres de panneaux ornés de la paroi gauche, tels qu'ils pouvaient apparaître aux yeux des artistes préhistoriques. Après avoir éliminé les destructions modernes sur les photographies des parois, on a comblé les lacunes de matière en clonant des états de surface les plus proches (fig. $\mathbf{n}^{\circ} 7$ ). Le support ainsi reconstitué, il fallait replacer les figures et les signes dans leur état au Magdalénien. La décision fut prise de figurer les gravures en blanc, telles qu'elles devaient apparaître lorsqu'elles étaient fraîchement incisées sur le calcaire. En partant des relevés des tracés préhistoriques, on a restitué les parties endommagées. Dans certains cas, il s'agissait simplement de compléter des aplats de couleur (par exemple le 
corps d'un grand bison ou l'axe d'un signe barbelé...) ou des contours gravés (crinière d'un cheval, cornes d'un bison...). Parfois, la présence de vestiges ténus de pigment noir et leur dispersion (notée sur le relevé) montraient que la peinture était tombée avec les écailles de roche qui la portaient : la comparaison avec d'autres figures mieux conservées dans la grotte fournissait alors la base de la restauration. Lorsque les figures étaient trop fragmentaires ou peu explicites (par exemple une créature indéterminée peinte en noir sous le ventre d'un cheval), nous les avons reproduites à l'identique. Enfin, pour un cheval et deux bisons gravés, on a pris le risque de « reconstituer » les têtes détruites (depuis la découverte ?) car il nous a semblé que leur mutilation actuelle ne rendait pas compte de leur état d'origine.

Figure 8
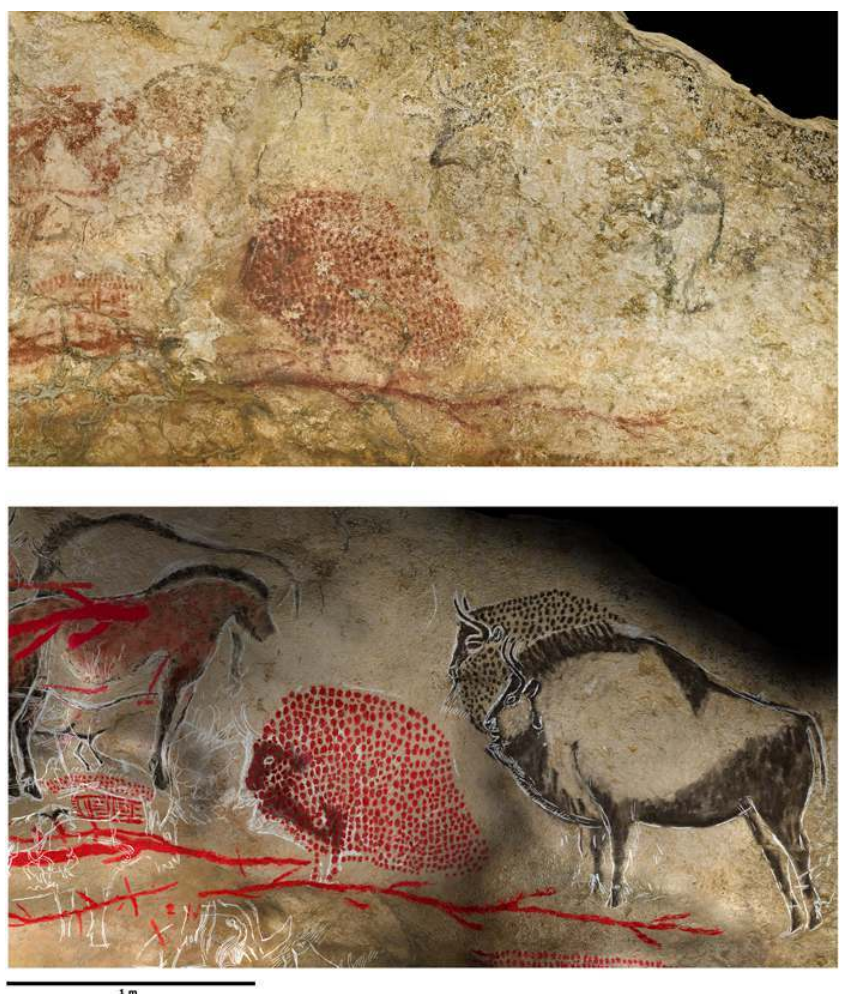

En haut, état actuel de la paroi ornée au centre du panneau principal (texturage de photos sur le modèle 3D). En bas, essai de restauration virtuelle des fresques dans leur état d'origine

Doc. Carole Fritz, Gilles Tosello. @ Ministère de la Culture et de la Communication, Carole Fritz, Gilles Tosello, Passé Simple, Cabinet Perazio

En effet, le naturalisme détaillé qui caractérise les corps de ces animaux nous a rappelé d'autres figures de Marsoulas de même facture; aucune d'entre elles n'étant privée de tête, il nous a semblé plausible d'en restituer une aux trois spécimens en question par clonage et mise à l'échelle. Ce parti pris, certes contestable, a montré aussi tout l'intérêt de cette démarche de "restauration ", non seulement pour le public mais aussi pour les spécialistes. Elle nous a fourni l'opportunité de tester les limites de notre travail, dans des directions où le chercheur n'aime guère s'aventurer. Par exemple, nous avons été surpris par la vigueur des couleurs, par le contraste entre les rouges et les noirs qui nous montrent l'art de Marsoulas sous un jour inhabituel, à peine crédible pour qui voit le site aujourd'hui; et pourtant, lorsque les couleurs étaient encore humides, les contours fraîchement gravés, il est probable que les fresques étaient aussi éclatantes que sur les 
images en 3D (fig. $\mathbf{n}^{\circ} \mathbf{8}$ ). Souvenons-nous des bisons du plafond d'Altamira dont les couleurs avaient tellement choqué les préhistoriens... de la fin du XIX ${ }^{\mathrm{e}}$ siècle.

\section{Conclusion}

18 Le premier bilan de cette expérience pluridisciplinaire, qui se situe au carrefour de compétences variées, est très positif. Bien sûr, la « restauration » réalisée virtuellement à Marsoulas n'est qu'une première tentative mais elle permet à chacun, spécialiste ou profane, en effaçant les outrages du temps, d'avoir une vue d'ensemble sur l'art pariétal du site, certainement plus proche de la réalité préhistorique que les vestiges qui en subsistent aujourd'hui. Un site web consacré à Marsoulas montre un extrait du film 3D en $\operatorname{ligne}^{13}:$ http://www.creap.fr/Marsoulas-3D.htm.

19 La 3D fournit la base d'une restitution de l'espace souterrain et des fresques qui peuvent être d'un grand intérêt pour la diffusion vers le public mais aussi pour la recherche. Dans l'avenir, nous souhaiterions parachever la visite virtuelle en ajoutant les secteurs ornés de la seconde moitié de la grotte, où la progression est la plus pénible.

Figure 9

\section{inp Institut national}

Communication présentée lors du séminaire ART RUPESTRE : LA 3D UN OUTIL DE MÉDIATION DU RÉEL INVISIBLE ? qui s'est tenu du 4 au 6 juin 2008 à Angles-sur-l'Anglin, coordonné par l'Institut national du patrimoine, avec la participation de la direction de l'architecture et du patrimoine

Pour compléter l'image globale de la cavité, il faudrait aussi s'intéresser aux sols. Les résultats de sondages archéologiques ont montré que la grotte avait connu une évolution géologique interne mouvementée, marquée par des effondrements de parois et l'action importante du ruisseau qui coule au fond. L'une des conséquences a été le remplissage progressif de la galerie, avec comme corollaire une modification de l'accès aux parois pour les artistes magdaléniens. Avec le même outil 3D, il serait intéressant de modéliser les différents niveaux de sols dans la grotte, en relation avec les hauteurs des panneaux ornés sur les parois. Ainsi, on pourrait aborder sous un nouvel angle la chronologie des événements à Marsoulas au cours des quinze derniers millénaires. (fig. $\mathbf{n}^{\circ}$ 9)

\section{NOTES}

1. En France, la grotte sous-marine Cosquer (Bouches-du-Rhône) fut la première grotte ornée scannée en 3D en 1991, grâce au mécénat d'EDF. Depuis, on peut citer, entre autres, les abris sculptés de la Chaire-à-Calvin (Charente) et du Roc-aux-Sorciers (Vienne), les grottes de Lascaux (Dordogne), Chauvet (Ardèche), Colombier (Ardèche), La Garma (Cantabria), La Peña de Candamo (Asturias)... 
2. Cau-Durban, Dominique. La Grotte de Marsoulas. Matériaux pour l'Histoire de l'Homme, 1885, $19^{\mathrm{e}}$ année, $3^{\mathrm{e}}$ série, II, p. 341-349.

3. Regnault, Félix. Peintures de Marsoulas. Bulletin de la Société Archéologique du Midi de la France, séance du 18 mai 1897, p. 127.

4. Cartailhac, Émile, Breuil, Henri. Les peintures et gravures murales des cavernes pyrénéennes : II, Marsoulas, près Salies-du-Salat (Haute-Garonne). L'Anthropologie, 1905, 16, p. 431-444.

5. Breuil, Henri. Marsoulas. In 400 siècles d'art pariétal : les cavernes ornées de l'Âge du Renne, Montignac : Éditions F. Windels, 1952, p. 239-245.

6. Plénier, Aleth. L'art de la grotte de Marsoulas. Mémoires de l'Institut d'art préhistorique, 1971, I, Toulouse.

7. Fritz, Carole, Tosello, Gilles. Marsoulas: une grotte ornée dans son contexte culturel. In Lejeune, M., Welté, A.C. (dir.). L'art du Paléolithique supérieur, XIV ${ }^{\mathrm{e}}$ congrès de l'Union Internationale des Sciences Pré-et Protohistoriques, Liège, 2001, ERAUL, Université de Liège, 2004, p. 55-68.

8. Fritz, Carole, Tosello, Gilles. Entre Périgord et Cantabres : les Magdaléniens de Marsoulas. In Jaubert, J., Barbaza, M. (dir.). Territoires, déplacements, mobilité, échanges durant la Préhistoire, 126e congrès national des sociétés historiques et scientifiques, Toulouse, 2001. Paris: Éditions du CTHS, 2005, p. 311-327.

9. Fritz, Carole, Tosello, Gilles. The hidden meaning of forms: methods of recording paleolithic art. Journal of Archeological Method and theory, 2007, vol. 24, n 1, p. 48-80.

10. Aujoulat, Norbert. Contribution de la saisie tridimensionnelle à l'étude de l'art et de son contexte physique. In Recherches pluridisciplinaires dans la grotte chauvet. Actes de la séance de la Société Préhistorique Française, 11 et 12 octobre 2003 à Lyon, éditions de la Société Préhistorique Française, 2005, Travaux 6, p. 189-198.

11. Missions réalisées en 2003, 2004 et 2006 par le cabinet de géomètres experts Guy Perazio. Y ont participé Guy Perazio, José Péral, Lionel Guichard (photographie), Carole Fritz et Gilles Tosello. Financement : Ministère de la Culture et de la Communication, DRAC Midi-Pyrénées.

12. Voir également dans ce même numéro : Fritz, Carole, Tosello, Gilles, Perazio Guy, Péral, José, Guichard, Lionel. Technologie 3D et relevé d'art pariétal : une application inédite dans la grotte de Marsoulas. [Document électronique]. Paris : Ministère de la Culture et de la Communication.

13. "Marsoulas, la grotte oubliée », film de 26 minutes produit par CNRS Images, France 3 Sud, Passé Simple, réalisé par Marc Azéma, écrit par Marc Azéma, Carole Fritz et Gilles Tosello. Un extrait en ligne est disponible sur le site : http://www.creap.fr/Marsoulas-3D.htm. Voir aussi : http://www.creap.fr/Marsoulas.htm.

\section{RÉSUMÉS}

Les techniques de scanner 3D sont appliquées dans des grottes et abris préhistoriques ornés avec un succès croissant. L'imagerie 3D peut même aider à restaurer les fresques des cavernes endommagées par le temps et les hommes. Pour la première fois, la "restauration virtuelle » d'une galerie ornée a été réalisée dans la grotte de Marsoulas; cette opération n'a pu être accomplie qu'en combinant la technologie 3D aux résultats de l'étude scientifique du site et du relevé des parois ornées. 
The techniques of 3D scanning are being applied in prehistoric caves and rock-shelters with increasing success. 3D imaging can even help restore the paintings of caves damaged by weathering or by human interventions (graffiti). For the first time, the 'virtual restoration' of a decorated gallery was undertaken in the cave of Marsoulas. This operation required the combination of 3D technology with the results of the scientific study of the site and the decorated walls.

\section{INDEX}

Mots-clés : art pariétal, grotte, Marsoulas, peinture, gravure, paléolithique, signe, 3D, scanneur, restauration virtuelle, modélisation, relevé

Keywords : virtual restoration of Paleolithic cave painting the example of the Marsoulas cave, cave art, cave, prehistoric painting, engraving, Paleolithic, pattern, scanner, tracing, virtual restoration, digital modelling

\section{AUTEURS}

CAROLE FRITZ

Chargée de recherche au CNRS, CREAP - Cartailhac, UMR 5608 TRACES. fritz@univ-tlse2.fr

\section{GILLES TOSELLO}

Chercheur associé, CREAP - Cartailhac, UMR 5608 TRACES. gilles.tosello@wanadoo.fr

\section{MARC AZÉMA}

Chercheur associé, CREAP - Cartailhac, UMR 5608 TRACES. marc.azema@wanadoo.fr

\section{OLIVIER MOREAU}

Infographiste 3D, Montpellier. contact@olivier-moreau.com

\section{GUY PERAZIO}

Cabinet de géomètres experts Perazio.gp@perazio.com

\section{JOSÉ PÉRAL}

Cabinet de géomètres experts Perazio 